\title{
Modelo de suprimento sanguíneo do intestino delgado e grosso da preguiça de coleira (Bradypus torquatus)
}

\author{
Blood supply model of the small and large intestine of the maned three-toed sloth \\ (Bradypus torquatus)
}

\author{
Jussara Rocha Ferreira' ${ }^{I}$ Lorenna Cardoso Rezende ${ }^{I I}$ Phellip Carvalho ${ }^{\text {III }}$ \\ Amanda Rocha Mortoza ${ }^{\text {III }}$ Daniele dos Santos Martins ${ }^{\text {II }}$
}

RESUMO

O Bradypus torquatus, conhecido como preguiça de coleira, está classificado na lista vermelha da IUCN como vulnerável. Este animal é um folívoro estrito que se alimenta de um pequeno número de plantas. O suprimento sanguíneo do intestino delgado e grosso de oito Bradypus torquatus, pertencentes ao acervo da Anatomia dos Animais Domésticos e Sivestres da Faculdade de Medicina Veterinária e Zootecnia da Universidade de São Paulo, foi estudado. O método incluiu preparação de relatório macroscópico, perfusão do sistema arterial com água $\left(40^{\circ} \mathrm{C}\right)$, injeção de látex corado (Neoprene ${ }^{\circledR}$ 650, Sulvinil ${ }^{\circledR}$ 2350-0003), fixação com formaldeido (10\%), conservação em solução modificada de Laskowiski e dissecação por mesoscopia de luz (Lupa LTS ${ }^{\circledR}$ 3700). A irrigação dos intestinos delgado e grosso dependeu da aorta abdominal, cujo ramo visceral ventral identificado como artéria mesentérica comum distribuiu-se no mesentério e mesocólon. Uma sequência de 9 a 25 ramos colaterais primários craniais destinaram-se ao duodeno, jejuno, íleo e parte da bolsa cecal. Outra sequência de 4 a 11 ramos caudais destinaram-se à bolsa cecal e cólons. No animal adulto, o modelo de vascularização do intestino diferiu dos outros vertebrados recentes em razão de não ocorrer coalescência peritoneal ao longo do intestino delgado e grosso.

Palavras-chave: angiologia, artéria mesentérica, Bradypodidae, tubo digestório.

\section{ABSTRACT}

The Bradypus torquatus also known as maned three-toed sloth is listed as vulnerable in the IUCN red list. This species is strict folivorous that feeds on a relatively small number of food plants. The blood supply in the small and large intestines of eight Bradypus torquatus was studied. These animals are from the Anatomia dos Animais Domésticos e Silvestres collection of the Universidade de São Paulo. The method included preparation of the macroscopic collection report, perfusion of the arterial network with water $\left(40^{\circ} \mathrm{C}\right)$, injection of colored latex (Neoprene $650^{\circledR}, 2350-0003$ Suvinil $^{\circledR}$ dye), fixation in formaldehyde (10\%), preservation in modified Laskowski solution and dissection under mesoscopic light (Lupa LTS ${ }^{\circledR}$ 3700). The blood supply of small and large intestine depends on the abdominal aorta, whose ventral visceral branch identified as the common mesenteric artery was distributed in the mesentery and mesocolon. A sequence of 9 to 25 primary collateral branches cranial is allocated to the duodenum, jejunum, ileum and part of the cecum. Another sequence of 4 to 11 caudal branches are destined to the cecum and colon pouch. The vascular pattern of adult maned three-toed sloth intestine differs from those of other previously described vertebrates, because there is no occurrence of coalescence peritoneal through small and large intestine.

Key words: angiology, mesenteric artery, Bradypodidae, digestive tract.

\section{INTRODUÇÃO}

Estudar animais da família Bradypodidae, no que diz respeito à sua morfologia, significa voltar na história da evolução dos desenhos corporais. Chamou a atenção o trato digestório, no qual parte do intestino terminal, o reto e o canal anal, projetaramse na região perineal, abrindo-se no trígono anal. Segundo a literatura, é difícil a sexagem nesses animais (GOFFART, 1971; MCCRANE, 1966; MARTINS, 2003) e, além disso, STEVENS \& HUME (1995) reconheceram no Myrmecophaga tridactyla um intestino delgado sete vezes maior que

${ }^{\mathrm{I}}$ Faculdade de Medicina, Área de Morfologia, Universidade de Brasília (UnB), 70735-000, Brasília, DF, Brasil. E-mail: jussararocha@unb.br. Autor para correspondência.

IIFaculdade de Medicina Veterinária e Zootecnia (FMVZ), Departamento de Clínica, Universidade de São Paulo (USP), São Paulo, SP, Brasil.

IIIFaculdade de Medicina, UnB, Brasília, DF, Brasil. 
o corpo deste tamanduá e, no Dasypus sabanicola, 0,825 vezes maior que o corpo deste tatu, enquanto DIZ et al. (2006) descreveram, em Chaetophractus villosus, um valor de 0,91 vezes. STEVENS \& HUME (1995) registraram, em Ursus americanus, um tubo intestinal medindo o equivalente a dez vezes o comprimento do corpo. GRASSÉ (1965), ao tratar da diferenciação e evolução do tubo digestório, reportou-se à existência de uma artéria comum ou celíaco-mesentérica em Heptranchias, e que nos répteis não existe correlação entre o comprimento do intestino e o regime alimentar. Entre os vertebrados, algumas classes apresentaram intestino pós-pilórico mais complexo, inclusive com o ceco desenvolvido. No coelho (Oryctolagus), o autor destacou uma grande artéria nutridora que invadiu os mesos dorsal e ventral (GRASSÉ, 1965).

Neste estudo, analisou-se o modelo arterial dos intestinos delgado e grosso da família Bradypodidae para fins de comparação de aspectos morfológicos e evolutivos entre o Bradypus torquatus e outros mamíferos.

\section{MATERIAL E MÉTODOS}

Cinco machos e três fêmeas de preguiça da espécie Bradypus torquatus (Illiger, 1811), família Bradypodidae, que vieram a óbito natural na Reserva Zoobotânica Matinha, localizada em Ilhéus/Bahia, foram doados para o acervo da Anatomia dos Animais Domésticos e Silvestres da Faculdade de Medicina Veterinária e Zootecnia da Universidade de São Paulo com registro do IBAMA n. 02006.001.348/98-31. Para análise dos animais, primeiramente, preparouse o relatório macroscópico de coleta (Tabela 1). O método incluiu a canulação da aorta torácica, perfusão com água $\left(40^{\circ} \mathrm{C}\right)$, injeção de látex corado (Neoprene ${ }^{\circledR}$
650; Sulvinil $\left.{ }^{\circledR} 2350-0003\right)$, fixação com formol (10\%), conservação em solução modificada de Laskowski, dissecação por mesoscopia de luz (Lupa LTS $3700^{\circledR}$ ), esquematização e foto-documentação (câmera Nikon $\left.\mathrm{D}-40^{\circledR}\right)$. A terminologia anatômica obedeceu aos critérios da NOMINA ANATOMICA VETERINARIA (2005), acrescidos de termos usuais contemplados na literatura, em razão da sua inexistência na lista oficial: bolsa cecal, artéria cólica distal, artéria ileocecal (aic), artéria cecocólica (acc), artéria arqueada (aar), artéria mesentérica comum (amco).

\section{RESULTADOS E DISCUSSÃO}

Ao analisar a irrigação dos intestinos de animais da família Bradypodidae, fez-se considerações sobre aspectos gerais da topografia das alças intestinais e dependências peritoniais que sustentam as vísceras e os elementos vásculonervosos na posição espacial, dando ênfase à origem, distribuição, tipo de ramificação e o modo de terminação dos vasos nutridores ao longo de seus trajetos.

Para o B. torquatus estudado, o comprimento em média do intestino delgado foi 170 centímetros $(\mathrm{cm})$, o intestino grosso $38 \mathrm{~cm}$ e o mesentério comum $22 \mathrm{~cm}$. O peritônio, ao fixar as alças intestinais, não coalesceu à parede abdominal, destacando-se do dorso do animal na linha mediana, como uma macromembrana dupla que, em sua borda de reflexão, envolveu as alças intestinais. Os intestinos delgado e grosso estiveram separados entre si, pela presença de uma dilatação, a bolsa cecal, com duas aberturas reduzidas, uma ileocecal e outra cecocólica. Bolsas cecais foram referidas por DIDIO et al. (1999) em vertebrados, semelhante ao encontrado no B. torquatus.

Tabela 1 - Registro, procedência, sexo $($ macho $=\mathrm{M}$, fêmea $=\mathrm{F})$, idade, peso, data do óbito e medidas corporais das preguiças $\boldsymbol{B r a d y p u s}$ torquatus provenientes da reserva Zoobotânica Matinha, localizada em Ilhéus/BA.

\begin{tabular}{|c|c|c|c|c|c|c|}
\hline \multirow{2}{*}{$\mathrm{N}-\underline{O}$ registro } & \multirow{2}{*}{ Procedência } & \multirow{2}{*}{ Sexo Idade } & \multirow{2}{*}{ Peso $(\mathrm{kg})$} & \multirow{2}{*}{ Data do Óbito } & \multicolumn{2}{|c|}{-------Segmento Corporal $(\mathrm{cm})-----$} \\
\hline & & & & & Corpo & Cauda \\
\hline 37 & Santa Luzia, BA & M. adulto & 4,70 & $01 / 02 / 00$ & 47,0 & 6,0 \\
\hline 65 & Almadina,BA & M. adulto & 4,00 & $06 / 01 / 00$ & 52,0 & 6,0 \\
\hline 99 & Jussari ,BA & M. adulto & 5,50 & $02 / 04 / 01$ & 42,0 & 4,0 \\
\hline 61 & Ubaítaba,BA & F. jovem & 4,05 & $06 / 12 / 99$ & 48,0 & 5,0 \\
\hline 92 & Itabuna,BA & F. adulta & 5,70 & $16 / 03 / 01$ & 62,0 & 8,0 \\
\hline 63 & Uruçuca,BA & F. jovem & 3,00 & $04 / 05 / 00$ & 48,0 & - \\
\hline 109 & Buerarema,BA & M. jovem & 3,90 & $05 / 11 / 01$ & - & 8,0 \\
\hline 66 & Itabuna,BA & M. jovem & 4,00 & $15 / 04 / 00$ & - & - \\
\hline
\end{tabular}

Ciência Rural, v.43, n.6, jun, 2013. 
Para vascularizar os intestinos do $\boldsymbol{B}$. torquatus, emergiu da aorta abdominal, um ramo visceral ventral ímpar, originado caudalmente à artéria celíaca, a amco. Sua origem, trajeto, subdivisões e terminação compuseram quatro desenhos básicos, esquematizados na figura 1. No esquema tipo 1 (casos $1 \mathrm{M}, 2 \mathrm{M}$ e $5 \mathrm{~F}$ ), a amco apresentou-se como um único vaso que terminou em anastomose arqueada com a primeira artéria cólica ventral. No tipo 2 (casos $4 \mathrm{~F}$ e 7M), a amco terminou por trifurcação em ramos ileocecal, cecal e cecocólicos. Ao longo do leito arterial, foram detectadas ilhas artérias, vasos que se anastomosaram formando um circuito arterial. No tipo $3(3 \mathrm{M}$ e $6 \mathrm{~F})$, a amco terminou por anastomose com artérias cecocólicas. Ao longo do vaso principal, encontrou-se um leito arterial paralelo na região do mesocólon com mais de uma derivação a partir da amco. No tipo 4 (8M), a amco apresentou-se relativamente retilínea e terminou por bifurcação em artérias ileocecal e cecocólica, sendo que esses dois vasos anastomosaram-se por uma artéria arqueada justa-cecal.

Os ramos da artéria mesentérica comum vascularizaram todos os seguimentos intestinais: o duodeno, o intestino delgado, o ceco, os cólons e também o fígado. Os ramos craniais da amco, ao longo do mesentério comum, estão relatados na sequência e compõe os dados da tabela 2.

A Artéria hepática (ah) derivou-se diretamente da amco como o primeiro ramo colateral

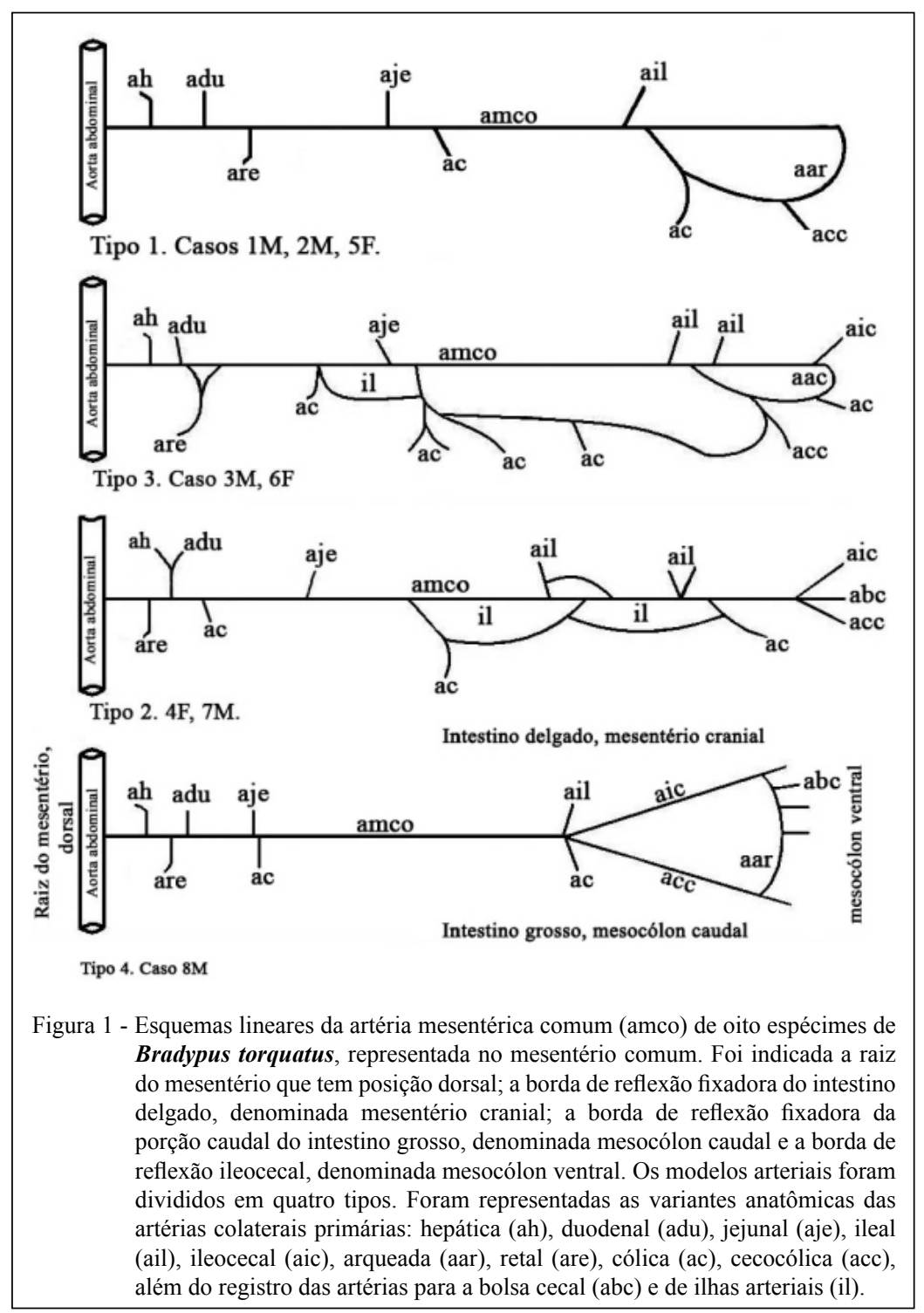

Ciência Rural, v.43, n.6, jun, 2013. 
Tabela 2 - Frequência absoluta dos ramos colaterais primários e secundários derivados da artéria mesentérica comum, por setor irrigado nos intestinos delgado e grosso da preguiça Bradypus torquatus, em função da amostra e sexo $($ macho $=\mathrm{M}$, fêmea $=\mathrm{F})$ e o modo de terminação dessas artérias.

\begin{tabular}{|c|c|c|c|c|c|}
\hline \multirow[t]{2}{*}{ Amostra (caso - sexo) } & \multicolumn{4}{|c|}{ 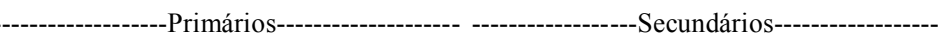 } & \multirow[t]{2}{*}{ Modo de terminação } \\
\hline & Mesentéricos & Cólicos & Íleocecais & Cecocólicos & \\
\hline $1-\mathrm{M}$ & 20 & 05 & 02 & anastomose & bifurca \\
\hline $2-M$ & 12 & 04 & 03 & 02 & bifurca \\
\hline $3-\mathrm{M}$ & 18 & 07 & - & - & anastomose \\
\hline $4-F$ & 17 & 11 & 02 & 01 & trifurca \\
\hline $5-F$ & 19 & 08 & - & - & anastomose \\
\hline $6-F$ & 25 & 07 & 02 & 01 & bifurca \\
\hline $7-\mathrm{M}$ & 21 & 09 & - & - & anastomose \\
\hline $8-\mathrm{M}$ & 09 & 07 & 06 & 04 & bifurca \\
\hline Total & 141 & 58 & 15 & 08 & \\
\hline Média & 17,62 & 7,25 & 1,87 & 1.0 & \\
\hline
\end{tabular}

primário cranial, ou indiretamente como ramo colateral cranial da primeira artéria duodenal.

As artérias duodenais (adu) foram ramos colaterais primários craniais da amco, com vasos retilíneos e curtos, devido à proximidade da alça jejunal em relação ao trajeto da artéria formadora, anastomosaram-se em arco com os vasos contralaterais ventrais. As subdivisões terminais da adu abordaram a parede do duodeno por intermédio de artérias retas de calibre reduzido, as quais bifurcaram na borda mesentérica da víscera, penetrando na sua intimidade.

As artérias jejunais (aje) e ileais (ail) são ramos colaterais primários dorsais da amco após a emergência da ultima artéria duodenal, topografia semelhante aos relatos de ramos da artéria mesentérica cranial verificados por MACHADO et al. (2002), e PEREIRA et al. (1978), em ratão do banhado e carneiro, respectivamente. As aje e ail craniais e caudais anastomosaram-se entre si, igualmente ao segmento de duodeno através de artérias arqueadas. Nesse setor, ocorreu maior quantidade de arcadas de segunda e terceira ordem, conforme relato de LLORCA(1952) em humanos e SOUZA et al. (2010) em tamanduá bandeira. Das arqueações, partiram ramos retos de menor calibre, organizados de forma que, ao aproximarem-se da borda mesentérica da alça intestinal, bifurcam-se, penetrando nesta pelos dois lados.

A artéria ileocecal (aic) representou um dos ramos da bifurcação, trifurcação ou anastomose terminal da amco (Figura 1, esquemas tipo 1 a 4 ). Essas artérias forneceram ramos colaterais dorsais arqueados, retos e oblíquos, dirigidos ao íleo terminal, e a bolsa cecal, além de ramos ventrais dirigidos ao peritônio. Os ramos colaterais distais da aic dirigidos à bolsa cecal foram oblíquos em sua origem e, em alguns casos, verificou-se formação de arcos secundários e terciários. A terminação da aic formou uma artéria arqueada na borda mesentérica da bolsa cecal. Esse vaso irrigou o compartimento cecal e anastomosou-se com as artérias cólicas (Figura 2).

Os ramos caudais da amco ao longo do mesocólon são destacados a seguir. As artérias retais (are) derivaram-se do vaso formador como o primeiro ou segundo ramo dorsoventral dirigidos ao intestino reto. A primeira artéria retal bifurcou-se, tendo seu ramo caudal margeado à alça intestinal e seu ramo cranial anastomosou-se em arco com a artéria contralateral, semelhante ao descrito para o intestino delgado. Ramos terminais retos abordaram a víscera de forma a irrigá-la a partir da região subserosa em direção à mucosa.

As artérias cólicas (ac) são ramos colaterais caudais da amco, relativamente paralelos e de fluxo em sentido oposto às aje e ail. A distribuição dos ramos cólicos colaterais se efetuou segundo a seguinte disposição geral: cada ramo se birfucou em ramos tranversais que anastomosaram-se entre si, formando arcos justaintestinais, dos quais derivaram-se os ramos terminais que atingiram perpendicularmente as alças cólicas. Em geral, ocorreram modificações segundo os diferentes seguimentos do tubo intestinal irrigado quanto ao número de artérias, o comprimento, a hierarquia de calibre e a arquitetura das arcadas justaintestinais (Figura 2).

A arquitetura espacial das artérias estudadas no B. torquatus foi muito particular. Sabe-

Ciência Rural, v.43, n.6, jun, 2013. 


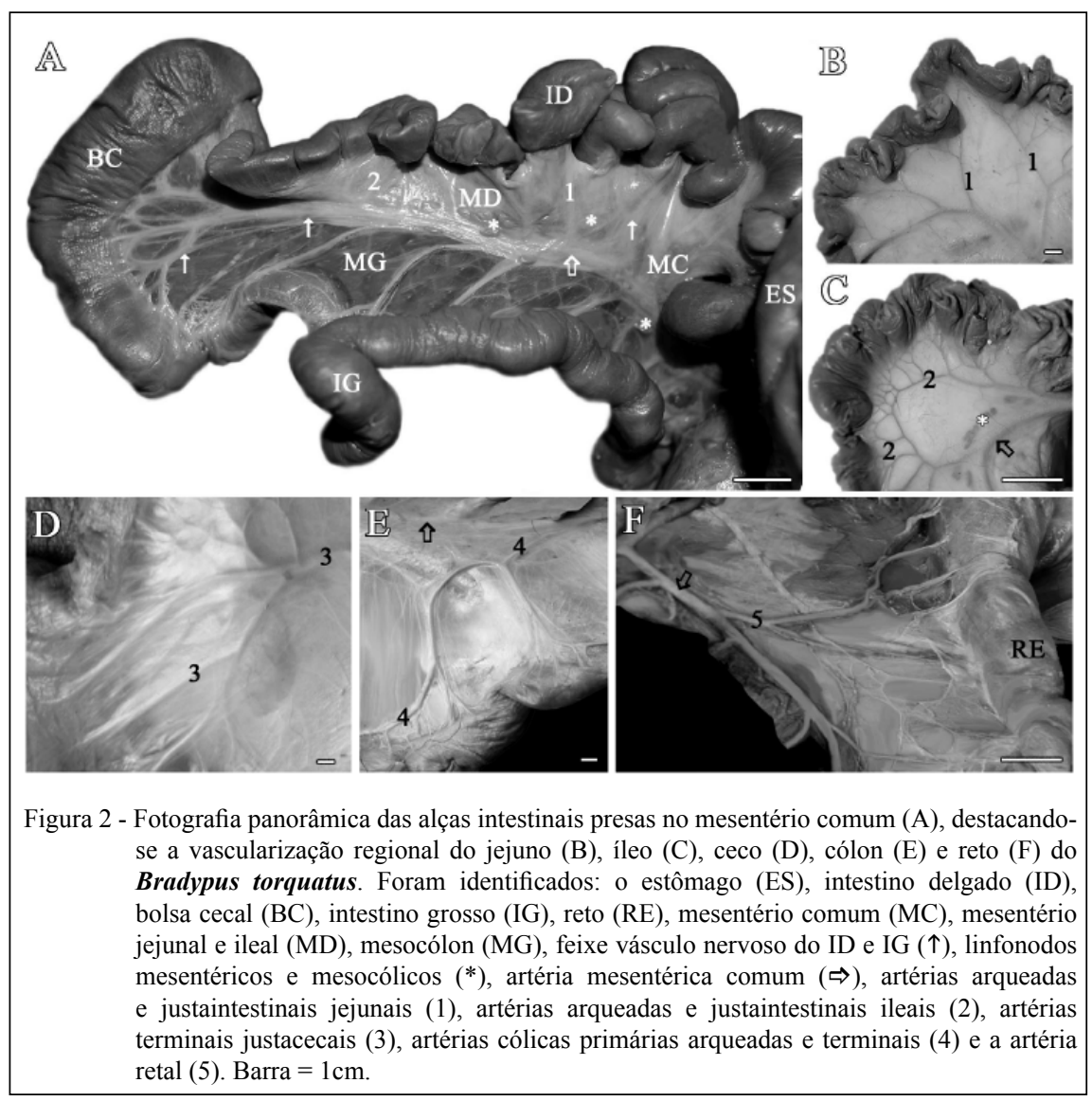

se que os vasos do tubo intestinal, intestinos médio e posterior, ontogeneticamente, derivam-se da aorta dorsal, emitindo dois ramos ventrais, as artérias mesentérica cranial (amcr) e caudal (amc). Estas, através do mesentério dorsal primitivo, distribuemse espacialmente dentro da cavidade peritoneal, atingindo as alças intestinais. Insinuadas ventralmente e indiferenciadamente no interior do saco vitelínico, são tracionadas pelo pedículo vitelínico e, caudalmente, em direção à cloaca, conforme sustenta a literatura (LLORCA, 1952; TESTUT \& LATARJET, 1960; GRASSÉ, 1965; JUNQUEIRA \& ZAGO, 1977; NODEN \& LAHUNTA, 1985; LAT SHAW, 1987; MOORE \& PERSAUD, 2008). $\mathrm{Na}$ maioria dos vertebrados adultos, a árvore arterial definitiva resultou do processo de desenvolvimento diferenciado das amcr e amc, em relação aos padrões de rotação da raiz do mesentério comum. Segundo SCHWARZE \& SCHRÖDER (1970), a raiz do mesentério comum, uma prega dupla em forma de bainha, em suas bordas, envolveria a alça intestinal específica, denominada mesentério para o intestino delgado e mesocólon para o intestino grosso. A afirmativa esteve coincidente com a proposta de outros autores (TESTUT \& LATARJET, 1960; GRASSÉ, 1965; NICKEL et al., 1977; DELLMANN \& BROWN, 1982; LAT SHAW, 1987) para os intestinos de vertebrados.

A amco do B. torquatus insinuou-se ventralmente, a partir do dorso do animal, em situação coincidente com a linha mediana, deslocando consigo o peritônio intestinal bilaminado. $\mathrm{O}$ vaso mediu entre 15 e $20 \mathrm{~cm}$ de comprimento. Todas as estruturas nervosas, o fígado, bem como os agrupamentos de linfonodos situados ao longo do trajeto da artéria e nas margens das alças jejunais e ileais foram nutridos exclusivamente pela amco e seus ramos colaterais e terminais.

No interior da cavidade abdômino-pélvica BROEK (1912), reconheceu modificações na serosa peritoneal em equidna, ornitorrinco, tamanduá e tatu. Essas diferenças tanto interferiram no padrão vascular das espécies que SCHWARZE \& SCHRÖDER (1970) comentaram que as amcr e amc insinuaramse pela lâmina própria do mesentério para atingir as alças intestinais em ruminantes, suínos e carnívoros e que suas topografias diferiram em razão do grau de rotação do peritônio.

Ciência Rural, v.43, n.6, jun, 2013. 
Os animais estudados exibiram um mesentério cuja raiz não se deslocou do plano sagital mediano, mantendo o vaso nutridor intestinal em posição intermediária entre as alças intestinais craniais (duodeno, jejuno e íleo), e pararelas a estas pelas alças intestinais caudais (bolsa cecal e os cólons), ou seja, o peritônio, ao se deslocar da borda ventral da aorta abdominal, levou consigo a amco. Não houve insinuação de outro vaso dirigido aos intestinos por entre as lâminas da serosa. Isso significa que a artéria nutridora do intestino terminal presente nos outros vertebrados, a artéria mesentérica caudal, não foi identificada no B. torquatus. Isso é indicativo de que, nesse estágio evolutivo, os graus de diferenciação e complexidade morfofuncional da rede arterial diferiram dos mamíferos recentes.

LLORCA (1952) demonstrou, no início do desenvolvimento do peritônio em humanos, fases rotacionais que lembraram o desenho definitivo da serosa e a posição topográfica da amco nos B. torquatus, ou seja, o peritônio nos humanos diferencia-se a partir de um modelo básico que, remotamente, na ontogenia, lembra o deste Xenarthra. No Myrmecophaga tridactyla, SOUZA et al. (2010) descreveram que o intestino grosso recebeu o sangue via artérias mesentéricas cranial e caudal, sustentadas por um mesentério comum, equivalente ao peritônio do B. torquatus.

Em carnívoros e herbívoros, BRUNI \& ZIMMERL (1947) registraram que a irrigação do tubo digestório derivou-se da grande amcr que anastomosou-se com as artérias cólica e mesentérica caudal. No B. torquatus, foram identificados, no trajeto da amco, anastomoses e ilhas arteriais, entre ramos cólicos e mesentéricos (Figura 2).

Coube destacar que a variação do número de artérias colaterais para o tubo digestório não interferiu na irrigação regional. Os ramos retais emergiram paralelamente aos hepatoduodenais e os ramos cólicos aos jejunoileais, havendo uma tendência para uniformizar o desenho vascular na região justaintestinal, pela presença dos vasos arqueados, equivalentes a outras descrições (MACHADO et al., 2006; CULAU et al., 2008; SOUZA et al., 2010).

Anastomoses arqueadas são facilitadoras do fluxo sanguíneo à montante ou à jusante da origem da artéria. Podendo contribuir para minimizar a falência vascular, visto que os vasos distribuem-se por pequenos segmentos. Isso foi recapitulado nesta série animal. Em primatas, o desenho arterial justaintestinal foi utilizado como um critério para diferenciar o jejuno do íleo (LLORCA, 1952; TESTUT \& LATARJET, 1960; MOORE \& PERSAUD, 2008). Em ungulados
(NICKEL et al., 1977) e carnívoros (GETTY, 1982; EVANS, 1993), diferenças na malha anastomótica justaintestinal também foram confirmadas.

$\mathrm{Na}$ região do intestino delgado, foram detectados de 09 a 25 ramos colaterais de primeira ordem no $\boldsymbol{B}$. torquatus e essa denominação foi admitida por TESTUT \& LARTARJET (1960) em humanos. O território vascular do intestino grosso exibiu de 4 a 11 artérias colaterais de primeira ordem, indicando potencial capacidade de anastomoses de irrigação nesse setor. Isto pode ser confirmado em outros estudos, independente da derivação do vaso nutridor (SILVA et al., 1985; CARVALHO et al., 1999; MACHADO et al., 2002; MACHADO et al., 2006; CULAU et al., 2008). Os pequenos vasos paralelos às bordas mesentéricas das alças, referidas por TESTUT \& LATARJET (1960) como justaintestinais (justacólicas, justaileais), que capilarizam-se na intimidade da parede, tiveram interpretações similares em outros estudos (NICKEL et al., 1977; GETTY 1982).

Quando o íleo conectou-se ao ceco, as artérias terminais tenderam a anastomosar-se em arcos justacecais. Isso significa dizer que, na zona de transição entre os intestinos delgado e grosso, existiu uma potencial capacidade de inversão de fluxo no caso de falência vascular no B. torquatus. Na maioria dos vertebrados descritos, o ceco foi nutrido pela artéria mesentérica cranial (SMITH \& MEADOWS, 1956; SMITH, 1959; NICKEL et al., 1977; PEREIRA et. al, 1978; BARNWAL et. al, 1982; GETTY, 1982; SILVA et al., 1985; MACHADO et. al, 2006).

\section{CONCLUSÃO}

Pode-se concluir que, no B. torquatus, o território vascular da artéria mesentérica comum apresentou-se independente e sem relação anastomótica com os sistemas arteriais próximos, parietal ou genitourinário, no que diz respeito à nutrição dos intestinos delgado e grosso. $\mathrm{Na}$ distribuição de seus ramos colaterais, a amco contribuiu com a maior parte da nutrição do fígado. De forma geral, cada ramo colateral se bifurcou em ramos relativamente tranversais, anastomosados entre si em arco. A não coalescência do peritônio à parede, conferiu aos intestinos delgado e grosso uma relativa fragilidade vascular em razão de impossibilitar a neoformação de vasos, no caso de obstrução da amco, por ausência completa de fluxo sanguíneo colateral. Os ramos arteriais que nutriram a bolsa cecal foram considerados análogos aos dos outros vertebrados recentes, visto que todos foram

Ciência Rural, v.43, n.6, jun, 2013. 
representações diversas de ramos caudais da primitiva artéria mesentérica cranial. $\mathrm{O}$ mesmo resultado não se pode dizer em relação aos cólons, visto que no $\boldsymbol{B}$. torquatus a artéria mesentérica caudal não existiu.

\section{AGRADECIMENTO}

Os autores agradecem ao Centro de Pesquisa da Lavoura Cacaueira (CEPLAC) em nome de Vera Lúcia de Oliveira, bióloga responsável pela Reserva Zoobotânica Matinha, localizada em Ilhéus/BA por doar para o acervo do departamento de Anatomia dos Animais Domésticos e Silvestres da Faculdade de Medicina Veterinária e Zootecnia da Universidade de São Paulo os animais objeto deste estudo.

\section{COMITÊ DE ÉTICA E BIOSSEGURANÇA}

O projeto foi aprovado pelo comitê de ética e bioética da Faculdade de Medicina Veterinária e Zootecnia da Universidade de São Paulo, protocolo n. 206/2002.

\section{REFERÊNCIAS}

BARNWAL, A.K. et al. Anatomical and roentgenographic studies on the cranial mesenteric artery of buffalo. Haryana Veterinary, v.21, n.1, p.1-5, 1982.

BRUNI, A.C.; ZIMMERL, V. Anatomia degli animali domestici. Milano: Francesco Vallardi, 1947. V.2, 763p.

BROEK, V.D. Zur frage der testicondie bei Bradypodidae Anatomischer Anzeiger, v.40, p.19-23, 1912.

CARVALHO, M.A.M. et al. Artérias mesentéricas cranial e caudal em cutias (Dasyprocta agouti). Veterinária Notícias, v.5, n.2, p.17-24, 1999.

CULAU, P.O.V. et al. Ramos colaterais viscerais da artéria aorta abdominal em Myocastor coypus (nutria). Acta Scientiae Veterinarie, v.36, n.3, p.241-247, 2008

DELLMANN H.D.; BROWN, E.M. Sistema digestivo. In: Histologia veterinária. Rio de Janeiro: Guanabara Koogan, 1983. p.164-294.

DIDIO, L.J. A. et al. Sistema digestório. In: __ Tratado de anatomia aplicada. São Paulo: Póllus, 1998. p.463-582.

DIZ, M.J.O. et al. Registro de medidas y pesos del tubo digestivo de un ejemplar de Chaetophractus villosus. Edentata, v.7, p.23-25, 2006. Disponível em: <http://www.bioone.org/doi/ abs/10.1896/1413-4411.7.1.23>. Acesso em 12 mar. 2012. doi: 10.1896/1413-4411.7.1.23.

EVANS, H.E. The heart and arteries. In: __. Miller's anatomy of the dog. Philadephia: Saunders, 1993. p.586-681.

GETTY, R. Anatomia dos animais domésticos. Rio de Janeiro: Guanabara Koogan, 1981. 1134p.

GOFFART, M. Function and form in the sloth. Oxford: Pergamon, 1971. 225p.

GRASSÉ, P.P. Traité de zoologie. Anatomie, systématique, biologie vertébres: genéralites embriologie topographique, anatomie compare. Paris: Masson et Cie Éditeurs, 1965. Tome XII, 1129p.

INTERNATIONAL COMMITEE ON VETERINARY GROSS ANATOMICAL NOMENCLATURE. Nomina anatomica veterinaria. New York: 2005. 166p. Disponível em: <http:// www.wava-amav.org/Downloads/nav 2005.pdf $>$. Acesso em: 12 mar. 2012

JUNQUEIRA, L.C.U.; ZAGO, D. Sistema alimentar e respiratório. In: . Fundamentos de embriologia humana. Rio de Janeiro: Guanabara Koogan, 1977. p.105-142.

LAT SHAW, W.K. Veterinary developmental anatomy: a clinically oriented approach. Missouri: Mosby, 1987. 283p.

LLORCA, F.O. Intestino médio. In: Anatomia humana. Barcelona: Científico médica, 1952. Tomo III, p.459-528.

MACHADO, G.V. et al. Suprimento arterial para as glândulas adrenais no ratão-do-banhado (Myocastor coypus Molina, 1782). Archives of Veterinary Science, v.7, n.2, p.9-14, 2002.

MACHADO, G.V. et al. Padrão de divisão e distribuição das artérias mesentéricas no ratão-do-banhado (Mycocastor coypus - Rodentia: Mammalia). Biotemas, v.19, n.1, p.59-63, 2006. Disponível em: $<$ http://www.biotemas.ufsc.br/volumes/pdf/volume191/p59a63. pdf $>$. Acesso em: 12 mar. 2012. ISSN: 0103-1543.

MARTINS, D.S. Morfologia do sistema reprodutor masculino da preguiça-de-coleira (Bradypus torquatus, Illiger, 1811). 2003. 116f. Dissertação (Mestrado em Ciências) - Programa de Pós-graduação em Anatomia dos Animais Domésticos e Silvestres, Universidade de São Paulo, SP.

MCCRANE, M.P. Birth, behaviour and development of a handreared two-toed sloth Choloepus didactylus. International Zoo Yearbook, v.6, n.1, p.153-163, 1966. Disponível em: <http:// onlinelibrary.wiley.com/doi/10.1111/j.1748-1090.1966.tb01733.x/ abstract>. Acesso em: 12 mar. 2012. doi: 10.1111/j.17481090.1966.tb01733.x.

MOORE, K.L.; PERSAUD, T.V.N. Embriologia clínica. 3.ed. Rio de Janeiro: Elsevier, 2008. 536p.

NICKEL, R. et al. The alimentary canal, general and comparative. In: _. Anatomy of the domestic mammals. 2 ed. Hamburg: Verlog Paul Parey, 1977. p.99-203.

NODEN, D.M.; LAHUNTA, A. The embryology of domestic animals. Developmental mechanisms and malformation. Baltimore: Williams e Wilkins, 1985. 367p.

PEREIRA, J.G.L. et al. A origem das artérias celíaca e mesentérica cranial, por tronco comum, em carneiros da raça corriedale. Revista de Medicina Veterinária e Zootecnia da Universidade de São Paulo, v.15, p.19-22, 1978.

SCHWARZE, E.; SCHRÖDER, L. El sistema visceral. In: Compendio de anatomia veterinária. Tomo II: El sistema visceral. Zaragoza: Acribia, 1970. p.11-118.

SILVA, F.O.C. et al. Contribuição ao estudo da origem e ramificação das artérias mesentéricas cranial e caudal, em fetos de bovinos azebuados. Brazilian Journal of Veterinary Research and Animal Science, v.22, p.31-36, 1985. 
SMITH, R.N.; MEADOWS, G.W. The arrangement of the ansa spiralis of the ox colon. Journal of Anatomy, v.90, p.523-526, 1956.

SMITH, R.N. The arrangement of the ansa spiralis of the goat colon. Anatomischer Anzeiger, v.106, p.101-103, 1959.

SOUZA, A.L.R. et al. Modelo de suprimento sanguíneo do intestino grosso do tamanduá bandeira (Myrmecophaga tridactyla). Ciência Rural, v.40, n.3, p.541-547, 2010. Disponível em: <http://
dx.doi.org/10.1590/S0103-84782010005000027>. Acesso em: 12 mar. 2012. doi: 10.1590/S0103-84782010005000027.

STEVENS, C.E.; HUME, I.E. Comparative physiology of the vertebrate digestive system. 2.ed. Cambrigde: Cambrigde University, 1995. 400p.

TESTUT, L.; LATARJET, A. Tratado de anatomia humana. Buenos Aires: Salvat, 1960. Tomo IV, 1168p.

Ciência Rural, v.43, n.6, jun, 2013. 